\title{
Assessment of activity of ocular sarcoidosis by gallium scanning
}

\author{
ANNI KARMA,' A ANNELI POUKKULA,'2 AND AIMO O RUOKONEN \\ From the Departments of 'Ophthalmology, 'Medicine, and 'Clinical Chemistry, University of Oulu, Oulu, \\ Finland
}

SUMmARY Gallium $\left({ }^{67} \mathrm{Ga}\right)$ citrate uptake over the orbits, parotid glands, and lungs was examined in six newly detected patients with sarcoidosis and 17 with chronic sarcoidosis. Six of $23(26 \%)$ had uveitis, 18/23 (78\%) decreased lacrimal secretion, and 13/16 (81\%) epithelioid cell granulomas in conjunctival biopsies. Ten patients with other diseases served as controls. Only five patients had ocular complaints and two had enlarged parotid glands. ${ }^{67} \mathrm{Ga}$ uptake over the orbits and parotids was measured by a quantitative computer based method. Gallium uptake was significantly higher over the orbits $(\mathrm{p}<0.001)$ and parotids $(\mathrm{p}<0.01)$ in the newly detected patients and in the parotids $(p<0.01)$ in the chronic group than the corresponding uptake in the controls. We suggest that in sarcoidosis an increased ${ }^{67} \mathrm{Ga}$ uptake in the orbital and parotid areas may be a phenomenon comparable to an increased ${ }^{67} \mathrm{Ga}$ uptake in the lungs in active alveolitis. Gallium scan is a good method for revealing even symptomless ophthalmic sarcoid changes. However, in chronic sarcoidosis an equal or only slightly increased gallium uptake over the orbits compared with background activity does not exclude ocular sarcoid disease.

Epithelioid cell granulomas are the histological hallmark of sarcoidosis. However, recent studies ${ }^{2}$ have shown that activation of immune cells precedes and modulates the formation of the granulomas. Studies with radioactive gallium $\left({ }^{67} \mathrm{Ga}\right)$ have added to the knowledge of the pregranulomatous cell mechanisms. In sarcoidosis ${ }^{67} \mathrm{Ga}$ has been shown to be taken up by 'activated' macrophages of pulmonary alveoli.' The studies concerning mainly pulmonary changes have also revealed that besides lung areas gallium accumulates often in localised extrathoracic sites, for example, in lacrimal and parotid glands. ${ }^{3-5}$

Only a few ophthalmological studies have attempted to clarify the phenomenon of gallium uptake over the orbito-parotid areas in sarcoidosis. Weinreb et al. " $"$ showed that, irrespective of an ocular sarcoid disease, ${ }^{67} \mathrm{Ga}$ accumulated in the lacrimal glands in sarcoidosis. Karma et al. ${ }^{\mathrm{k}}$ suggested that in ocular sarcoidosis confirmed by conjunctival biopsies both ocular and parotid areas accumulate radiogallium.

We examined gallium " $\mathrm{Ga}$ citrate uptake over the orbits, parotids, and lungs in patients with newly Correspondence to Anni Karma, MD. Suvantokatu 1 B 33, SF33100 Tamperc, Finland. detected untreated sarcoidosis and patients with chronic sarcoidosis with concurrent ophthalmic sarcoid changes. The intensity of the gallium activity was calculated by a semiquantitative computer based method.

\section{Materials and methods}

Twenty-three patients with generalised active sarcoidosis, six of recent onset and 17 chronic cases, and 10 controls were selected for the study. The diagnosis of sarcoidosis was based on a typical clinicoradiological picture and was confirmed histologically in every patient (by mediastinoscopy, biopsy of bronchial mucosa or skin, or by the Kveim test).

Group 1: Newly detected sarcoidosis. This group consisted of six patients whose sarcoidosis at the time of gallium scanning had been diagnosed less than 12 months previously. Five patients were referred by a chest physician for an ophthalmic examination because of marked gallium accumulation seen over the orbits and parotid areas. They had no ocular complaints, but two had enlarged parotid glands bilaterally. One patient (case 1) had a history of 
Table 1 Ophthalmic findings in sarcoidosis patients

\begin{tabular}{|c|c|c|c|c|c|c|}
\hline \multirow[t]{2}{*}{$\begin{array}{l}\text { Type of } \\
\text { sarcoidosis }\end{array}$} & $\begin{array}{l}\text { Positive } \\
\text { conjunctival } \\
\text { biopsy }\end{array}$ & \multicolumn{2}{|c|}{$\begin{array}{l}\text { Lacrimal } \\
\text { gland } \\
\text { disease* }\end{array}$} & \multicolumn{2}{|c|}{$\begin{array}{l}\text { Active } \\
\text { uveitis }\end{array}$} & \multirow[t]{2}{*}{$\begin{array}{l}\text { Total } \\
\text { no. of } \\
\text { patients } \\
\text { No. }\end{array}$} \\
\hline & $\%$ No. & & No. & $\%$ & No. & \\
\hline $\begin{array}{l}\text { Newly } \\
\text { detected } \\
\text { cases }\end{array}$ & $75 \quad(3 / 4)$ & 67 & $(4 / 6)$ & 33 & $(2 / 6)$ & 6 \\
\hline $\begin{array}{c}\text { Chronic } \\
\text { cases }\end{array}$ & $83(10 / 12)$ & 82 & $(14 / 17)$ & 23 & $(4 / 17)$ & 17 \\
\hline Total & $81 \quad(13 / 16)$ & 78 & $(18 / 23)$ & 26 & $(6 / 23)$ & 23 \\
\hline
\end{tabular}

${ }^{*}$ Assessed by Schirmer's I test.

neurological symptoms, weight loss, and malaise for three years. Her sarcoidosis, Heerfordt's syndrome, was of chronic type, though the diagnosis was delayed. All the patients had pulmonary sarcoid $x$ ray changes. Angiotensin converting enzyme (ACE) was increased in four patients. The mean ACE was 96.5 U/l (range 40-155 U/l, normal range 25-80 U/l). None had received oral or topical steroids (eye drops) by the time of the gallium scan.

A detailed ophthalmological examination included measurement of visual acuity, eye movements, biomicroscopy of the anterior segment of the eyes, measurement of ocular pressure, ophthalmoscopy, and three-mirror-glass examination of the peripheral fundus. Lacrimal secretion was examined by Schirmer I testing after preliminary biomicroscopy of the eyes. Lacrimal secretion was assessed as decreased when the length of the moistened test strip was $<7 \mathrm{~mm}$. A conjunctival biopsy specimen was taken from a suspicious nodule, if seen, otherwise from the normal looking conjunctiva of the lower fornix by the method described previously."' The sections were stained with haematoxylin-eosin. Epithelioid cell granulomas under an intact epithelium were interpreted as having been produced by sarcoidosis.

The ophthalmological examination of the newly detected patients revealed conjunctival granulomas in three out of four biopsied cases, decreased lacrimal secretion in four, and uveitis in two patients (cases 1 and 2) (Table 1).

Group 2: Chronic ocular sarcoidosis. This group consisted of 17 patients who, after a personal followup (by AK) of 10 years on the average, still had one or more of the following ocular sarcoid findings: conjunctival granulomas in 10 of the 12 biopsied cases, decreased lacrimal secretion in 14 , and active uveitis in four (Table 1). Only five patients had symptoms of their ocular sarcoid change: three of uveitis and two of decreased lacrimal secretion. Two patients with a negative conjunctival biopsy during the first years of the disease had developed conjunctival granulomatosis by the time of the present study. One patient with a positive conjunctival biopsy initially had no suspicious lesions in the conjunctivae at the present follow-up examination and the biopsy was negative. Four positive conjunctival biopsies disclosed fibrous tissue round the epithelioid cell aggregates (Fig. 1).

All the patients with a decreased lacrimal secretion considered to be of sarcoid origin during the time of the diagnosis of the disease still had it at the time of this study. Seven patients had initially had uveitis. In three of them uveitis had become inactivated by the time of this study. At the onset of the disease one
Fig. 1 Conjunctival sarcoidosis. Epithelioid cell granulomas surrounded by fibrous tissue in a patient with chronic sarcoidosis. Haematoxylin and eosin.

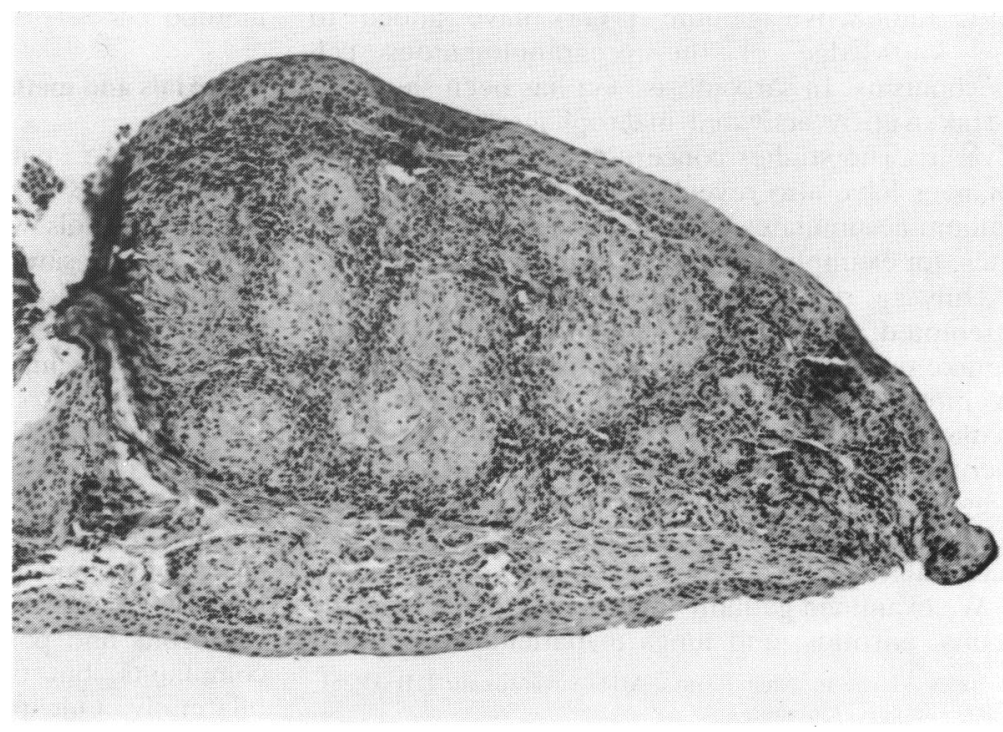


patient had had uveoparotid fever. The parotids had become normal in size within a year, but nine years later uveitis was still running a very active course. In addition two patients without uveitis but with conjunctival granulomatosis and lacrimal gland disease had had a transient enlargement of the parotid glands during the first months of the disease.

Either initially or some years after the onset of the disease all the patients had had pulmonary $x$-ray changes. In seven patients the $x$-ray changes had resolved by the time of the gallium scanning.

Twelve of the 17 patients in this group had increased or borderline ACE. The mean ACE was 83.5 U/l (range 16-184 U/l). In five patients with normal ACE the disease showed clinical activity: uveitis, enlarging skin lesions, or deterioration of the pulmonary function tests.

Ten patients had received oral prednisolone for two to 92 months, and seven patients had received topical steroids during the years of the follow-up. Three patients received dexamethasone eye drops at the time of the scanning, but none were on oral steroids.

Group 3: Controls. Gallium scan was also per-

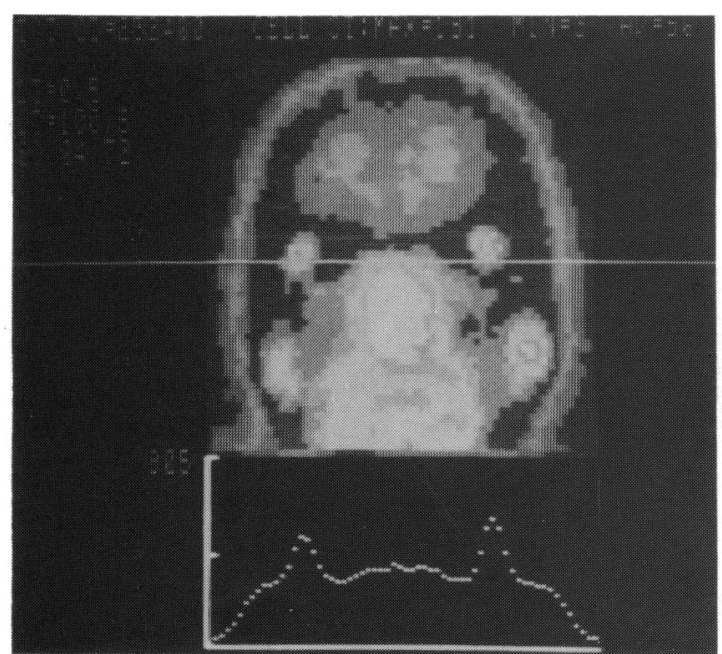

Fig. 2 Gallium ${ }^{67}$ Ga citrate uptake over the orbits in the anterior view of the head as seen in the gamma camera computer screen. The area round the horizontal line crossing the orbits was used for the construction of the activity profile by the computer. Height of the area was 2 or 3 pixel lines having the maximum activities (maximum number of counts per pixel) of the orbits. The activity profile (amount of activity in the vertical scale and location of the activity in the horizontal scale) is shown in the lower part of the figure. Gallium uptake over the orbits (the two peak activities of the profile) were expressed in percentages of the minimum background activity $(100 \%)$ of the profile between the orbits. Relative uptake of the parotid glands was calculated similarly from a horizontal activity profile crossing the parotid glands. formed on 10 control patients, seven with chronic bronchitis, asthma, or allergic alveolitis and three with ocular complaints (one with uveitis and two with suspicion of sicca syndrome). Sarcoidosis was excluded in all of them.

\section{SCANNING TECHNIQUE}

Each patient received $1.5 \mathrm{MBq} / \mathrm{kg}{ }^{67} \mathrm{Ga}$ citrate intravenously $48 \mathrm{~h}$ prior to scanning. Anterior views were obtained of the head, chest, and upper abdominal regions with a wide scanner (Siemens, Rota-Camera ZLC-75) and analysed by the Gamma-11 computer system. Using anterior views of the head the computer constructed two horizontal activity profiles of gallium uptake, one crossing the orbits (Fig. 2) and the other the parotid glands. The activity profiles comprised two or three vertical pixel lines having the maximum activity (maximum number of counts per pixel) of the orbits and the parotid glands respectively. Gallium uptake of the orbits and the parotid glands was expressed in percentages of the minimum background activities $(100 \%)$ of the profiles between the orbits and parotid glands respectively (Table 2 ).

Uptake in the lung hila and parenchyma was graded from 0 to 3 relative to liver activity (classification proposed by several investigators ${ }^{13}$ "I). Grade 0 signified uptake comparable to background activity; grade 1 uptake greater than background but less than liver activity; grade 2 uptake equal to, and grade 3 uptake greater than, liver activity.

Table $2{ }^{67}$ Ga uptake over the orbits, parotid glands and lungs in sarcoidosis patients and controls*

\begin{tabular}{|c|c|c|c|c|c|}
\hline \multirow{3}{*}{$\begin{array}{l}\text { Classification } \\
\text { of the groups }\end{array}$} & \multicolumn{5}{|c|}{ Gallium uptake* } \\
\hline & \multicolumn{2}{|l|}{ Orbits $(\%)$} & \multicolumn{2}{|c|}{ Parotids (\%) } & \multirow{2}{*}{$\begin{array}{r}\text { Lungs } \\
0123\end{array}$} \\
\hline & Meant $\pm s d$ & Range & Meant $\pm s d$ & Range & \\
\hline $\begin{array}{l}\text { Newly detected } \\
\text { sarcoidosis } \\
(n=6)\end{array}$ & $252 \pm 69 \S$ & $164-35$ & $5191 \pm 93 \neq$ & $100-418$ & 123 \\
\hline $\begin{array}{l}\text { Chronic } \\
\text { sarcoidosis } \\
(n=17)\end{array}$ & $146 \pm 27$ & $100-206$ & $145 \pm 38 \ddagger$ & $100-221$ & 1034 \\
\hline $\begin{array}{l}\text { Controls } \\
\qquad(n=10)\end{array}$ & $142 \pm 27$ & $112-17$ & $7104 \pm 10$ & $100-128$ & 10 \\
\hline
\end{tabular}

*Uptake of the orbits and the parotid glands was expressed in percentages of maximum activity (number of counts per pixel) out of the minimum background activity $(100 \%)$ in the horizontal activity profile between the orbits and parotid glands, respectively. Uptake by the lungs was graded $0-3$ relative to liver activity. Grade () signified uptake comparable to background activity; grade 1 uptake higher than background but less than liver activity; grade 2 uptake equal to, and grade 3 uptake higher than, liver activity.

†The average activity of the two orbits and two parotids, respectively.

$\ddagger$ Significantly $(\mathrm{p}<0 \cdot 01)$, \$highly significantly $(\mathrm{p}<0 \cdot(0) 1)$ different from the control value by Student's $t$ test. 


\section{Results}

The relative intensity of the gallium uptake over the orbits, parotids, and lungs in the three groups are shown in Table 2. The "7 Ga uptake over the orbits in the newly detected patients with sarcoidosis significantly exceeded the corresponding ${ }^{67} \mathrm{Ga}$ uptake in the chronic patients and controls $(\mathrm{p}<0.001$ by Student's $t$ test). The ${ }^{67} \mathrm{Ga}$ uptake of the parotid areas in both the newly detected $(p<0.001)$ and the chronic $(p<0.01)$ sarcoidosis patients significantly exceeded the corresponding activities in the controls. The difference in the values of the parotid uptake between the newly detected patients and the chronic ones was not significant $(p<0 \cdot 5)$. All the newly detected sarcoidosis patients showed an increased lung uptake (grade 1 to 3 ). In 10 patients of the chronic group and in all the controls the lung uptake was not increased. One patient in the newly detected group with an orbital uptake of $170 \%$ showed an uptake of $100 \%$ by the parotids. She had a bilateral hilar adenopathy on $x$ ray, erythema nodosum, and no sarcoid changes were found in the ophthalmological examination. The heaviest ${ }^{67} \mathrm{Ga}$ uptake by the parotids among the newly detected patients was seen in the patient with Heerfordt's disease (case 1). She had the lowest lung uptake in this group.

None of the chronic patients had a mean uptake over the orbits greater than the corresponding mean uptake in the new cases $(252 \%)$, but in four chronic patients the parotid uptake exceeded the corresponding mean uptake in the new cases $(191 \%)$.

In three patients gallium scanning was done twice. In one patient with a chronic sarcoidosis the scanning had been performed eight months before the present study, and in two new patients the scanning was repeated six months and eight months after the first procedure. These cases are briefly reported.

\section{CASE 1}

A 56-year-old woman had for three years experienced weight loss, malaise, increasing tremor and rigidity, and transient facial palsies. She was referred for an ophthalmological examination because of complaints of decreasing vision and constriction of the visual fields. The left facial nerve was weak and the submandibular and parotid glands were enlarged and warm on palpation. Her visual acuity was 20/100 in the right and 20/40 in the left eye. Lacrimal secretion was decreased to $1 \mathrm{~mm}$ in both eyes. Granulomatous bilateral uveitis was seen. Intraocular pressure was $30 \mathrm{mmHg}$ in both eyes, and the optic discs showed glaucomatous cuppings. Visual field examination disclosed marked defects compatible with glaucomatous changes. ACE was $124 \mathrm{U} / \mathrm{l}$. Lysozyme was $21 \mathrm{mg} / \mathrm{l}$ (normal range 3-9 mg/l). A tuberculin skin test to 100 tuberculin units was nonreactive. Chest $x$-rays showed parenchymal infiltrations. Conjunctival biopsy was negative. Biopsy of the skin lesion of the forehead showed noncaseating granulomatous inflammation. Sarcoidosis with a classical Heerfordt's syndrome was diagnosed. Gallium scan (Fig. 3) showed ${ }^{67} \mathrm{Ga}$ uptake to $223 \%$ over the orbits and $418 \%$ in the parotid areas respectively, but only slightly increased lung uptake (grade 1). With topical corticosteroids the uveitis became quiescent and the pressure became normal. Systemic corticosteroids had to be restricted because of their psychogenic side effects. Six months later the gallium images of the head and chest were unchanged.

CASE 2

A 37-year-old man with pulmonary sarcoidosis was referred to an ophthalmological examination because, in addition to an increased lung uptake (grade 3), an increased ${ }^{67} \mathrm{Ga}$ uptake of the orbital and parotid areas was seen (Figs. 4A, B). Mean gallium uptake over the orbits was $250 \%$ and that of the parotids $175 \%$, respectively. He had no ocular complaints. Visual acuity was $20 / 20$ in both eyes. Lacrimal secretion was decreased to $1 \mathrm{~mm}$ in the right and $5 \mathrm{~mm}$ in the left eye, and a peripheral uveitis was seen. Examination of the conjunctivae showed isolated yellowish nodules typical of sarcoidosis. On biopsy they were found to contain epithelioid cell granulomas. The patient received oral and topical

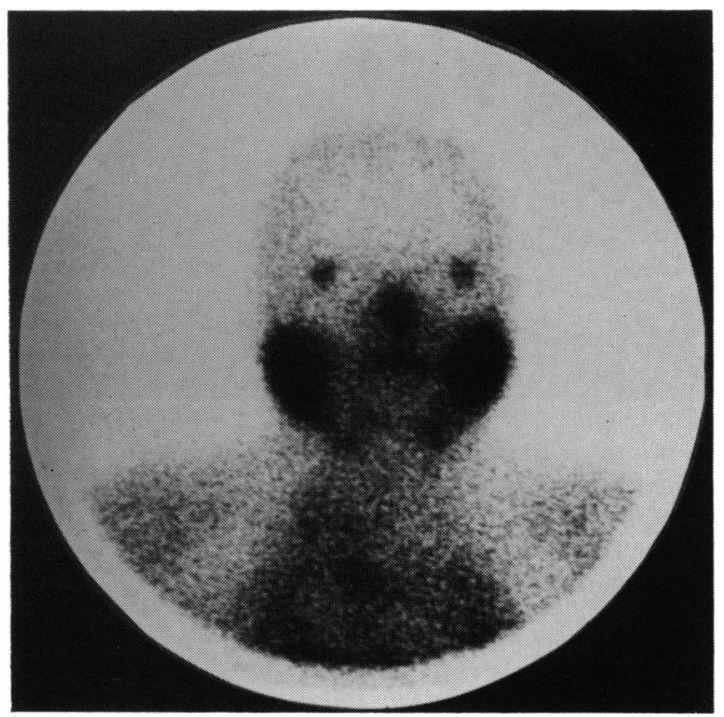

Fig. 3 Case 1. Newly detected sarcoidosis. Gallium scan of the head showing a highly increased ${ }^{67} \mathrm{Ga}$ uptake over the orbits $(223 \%)$ and parotid glands $(418 \%)$ in a 52 -year-old woman with a classical uveoparotid fever. 


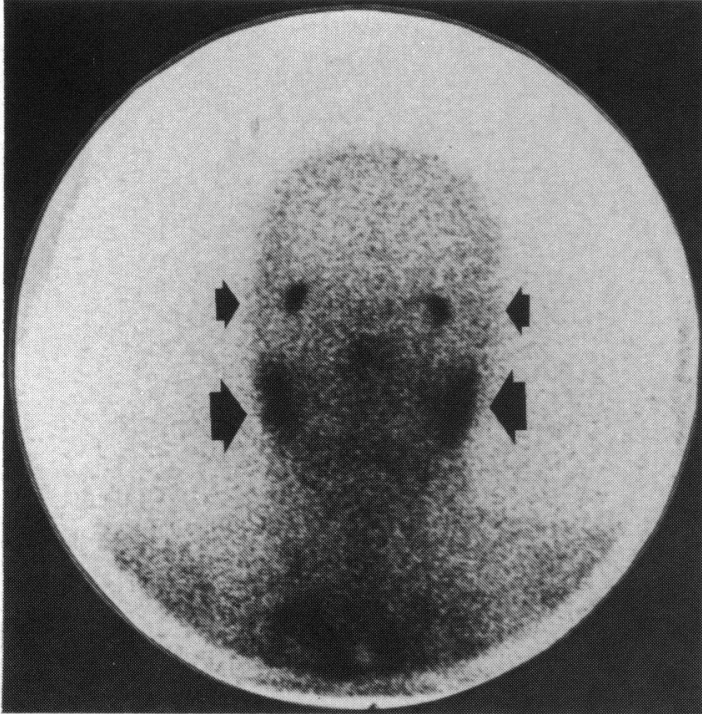

Fig. 4A

Fig. 4 Case 2. Newly detected sarcoidosis. Gallium scan showing an increased ${ }^{67}$ Ga uptake (A) over the orbits $(250 \%)$, parotids (175\%) (arrows), and (B) lungs (grade 3 ) (arrows) in a 30-year-old man with subclinical uveal, conjunctival, and lacrimal gland sarcoidosis. Thoracic contours are marked with dots. $L=$ liver.

corticosteroids for one month. In a follow-up examination six months later the lacrimal secretion became normal. There was no uveitis, but sarcoid nodules were still seen in the conjunctivae. The gallium images showed much decreased activity over the orbits (187\%) and parotids (114\%) and no activity in the lung areas (grade 0 ).

\section{CASE 3}

A 52-year-old woman had had in the 1950s a right parotidectomy because of malignant disease. Pulmonary sarcoidosis was diagnosed in July 1980. Since then she had been receiving oral prednisolone beginning with a dose of $20 \mathrm{mg} / \mathrm{day}$. ACE was initially $170 \mathrm{U} / \mathrm{l}$ but decreased to a normal range in three months. $X$-ray changes and pulmonary symptoms deteriorated in October 1982. Visual acuity was $20 / 40$ in the right eye and $20 / 25$ in the left eye, and a symptomless uveitis was seen in both eyes. Gallium scan showed an increased uptake over the orbits $(157 \%)$ and left parotid $(208 \%)$, but the lung uptake was not increased (grade 0) (Figs. 5A, B). $\mathrm{ACE}$ rose to $106 \mathrm{U} / \mathrm{l}$ after the prednisolone was discontinued in February 1983. Ophthalmic examination showed reactivation of uveitis bilaterally and nodes in the left iris (Fig. 6A). Lacrimal secretion had ceased ( $1 \mathrm{~mm}$ in both eyes). Conjunctival biopsy gave

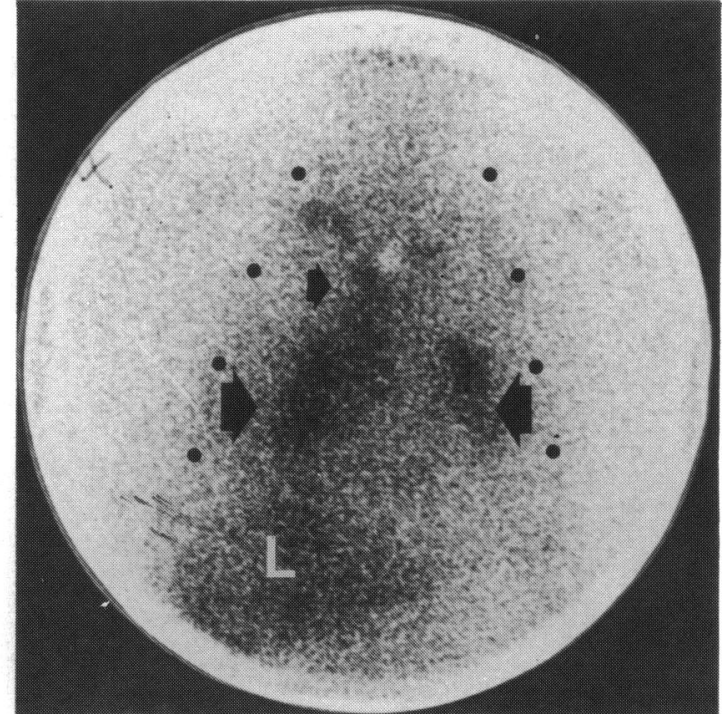

Fig. 4B

negative results. This time gallium images showed increased activity in lung areas (grade 3) (Fig. 6B), but the uptake over the orbits and the left parotid was of the same magnitude as eight months previously.

\section{Discussion}

Gallium scanning is a sensitive method for revealing active pulmonary sarcoidosis. ${ }^{1-5}$ But it is an unspecific method, for ${ }^{67} \mathrm{Ga}$ citrate has been shown to accumulate also in a wide variety of inflammatory and neoplastic thoracic and extrathoracic sites. ${ }^{12}{ }^{13}$ Gallium uptake over the orbits or parotids is not specific for sarcoidosis either. An increased uptake in these areas can be seen, for example, in Sjögren's syndrome, lymphomas, ${ }^{7}$ or even normally. ${ }^{1+}$ However, the combination of a concurrently increased gallium uptake by orbits, parotids, and lungs is a frequent phenomenon in sarcoidosis and has been considered characteristic of it. ${ }^{\text {3.-5 }} 11$

The present study was designed to evaluate the ${ }^{67} \mathrm{Ga}$ uptake in the orbito-parotid areas in newly detected and chronic sarcoidosis patients with clinical ophthalmic sarcoid findings. Quantitation of the intensity of ${ }^{67} \mathrm{Ga}$ uptake has been based on visual inspection of the images in most studies. ${ }^{1-5}$ Our method quantitated the intensity of gallium uptake over the orbital and parotid areas by a computer; this improved the accuracy of the evaluations and the comparability of the findings.

All but one patient in the newly detected group and all the chronic patients had one or more clinical ophthalmic sarcoid findings and $81 \%$ of all patients had a positive conjunctival biopsy. Gallium images 


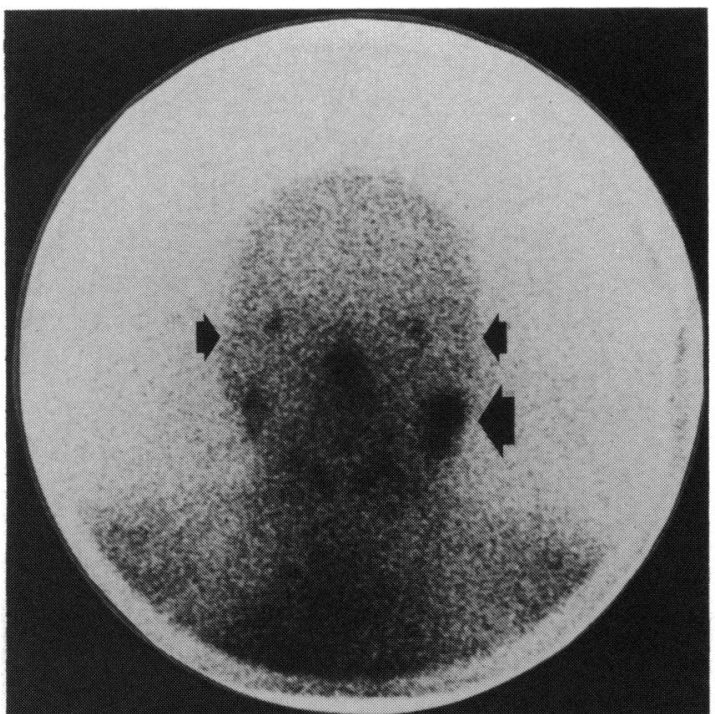

Fig. 5A

Fig. 5 Case 3. Chronic sarcoidosis. Gallium scan showing an increased ${ }^{67}$ Ga activity (A) over the orbits (157\%) and left parotid (208\%) (arrows) (right parotid resected), but not (B) in the lungs (grade 0), in a 52-year-old female with pulmonary and subclinical uveal sarcoidosis. Thoracic contours are marked with dots. $L=$ liver.

showed a highly significantly heavier uptake over the orbits in the newly detected patients and a significantly heavier parotid uptake of both the newly detected and chronic patients compared with the corresponding uptake in the controls. In addition all recently detected patients and two-fifths of the chronic patients showed an increased lung activity.

Bronchoalveolar lavage analyses have shown that in active pulmonary sarcoidosis ${ }^{67} \mathrm{Ga}$ is taken up by activated alveolar macrophages constituting a state of 'high intensity alveolitis', often with an increased number of activated T lymphocytes. ${ }^{2}$ It is probable that the increased ${ }^{67} \mathrm{Ga}$ accumulation over the orbits and parotids in sarcoidosis is due to an increased density of activated macrophages in these areas, being especially prominent in the newly detected untreated cases.

Because of selection of the cases it cannot be said how often an increased ${ }^{67} \mathrm{Ga}$ uptake in the orbital and parotid areas occurs in sarcoidosis patients. Koegh and associates ${ }^{2}$ have shown that in pulmonary sarcoidosis a state of low intensity alveolitis which includes, for example, a negative ${ }^{67} \mathrm{Ga}$ image of the lung areas (normal lung uptake) is four times more prevalent than a state of high intensity alveolitis with a positive lung image (increased lung uptake). The high intensity alveolitis in their patients was often

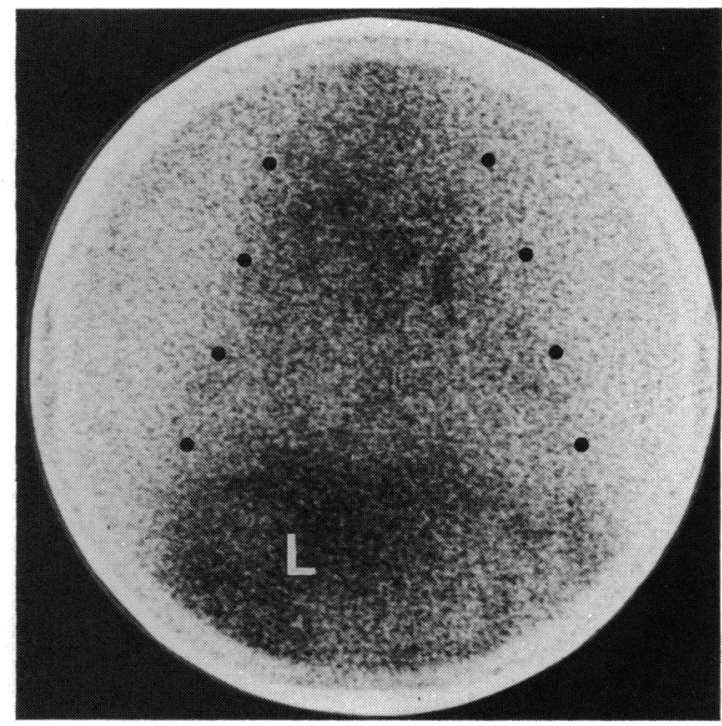

Fig. 5B

followed by a deterioration of the lung functions. They also showed that alveolitis could be unstable and often reverted from a high intensity state to a low intensity state and, more seldom, also vice versa. The decrease of ${ }^{67} \mathrm{Ga}$ activity over the orbits, parotids, and lungs in our patient no. 2 and an increase of the lung uptake in patient no. 3 (Figs. 5 and 6) are examples of such a reversion. In patient no. 2 recovery of lacrimal secretion was seen together with a decrease in ${ }^{67} \mathrm{Ga}$ uptake over the orbits and parotids. And, further, patient no. 3 showed reactivation of uveitis with iris granulomas in association of an increased lung uptake.

The lacrimal gland, a gland comparable to the parotid in its structure and function, is possibly the main locus responsible for the ${ }^{67} \mathrm{Ga}$ uptake over the orbits in sarcoidosis. Our study suggests that the lymphoproliferative tissues of the conjunctivae and possibly also the intraocular tissues can be a site of activated cell elements to be seen in gallium imaging.

In conclusion we suggest that an increased ${ }^{67} \mathrm{Ga}$ uptake over the orbits and parotids in sarcoidosis probably indicates activation of immune effector cells in these areas, and clinical sarcoid involvement, often asymptomatic, may be revealed on ophthalmic examination. On the other hand, in chronic sarcoidosis a negative gallium image or only a slightly increased ${ }^{67} \mathrm{Ga}$ uptake over the orbits or parotids does not exclude clinical sarcoid ocular disease or granulomatous change in conjunctival specimens.

Gallium findings support the results of ophthalmological examinations ${ }^{1516}$ and of sialographic studies $^{17}$ and indicate that in sarcoidosis the lacrimal glands, conjunctivae, and parotid glands are affected 


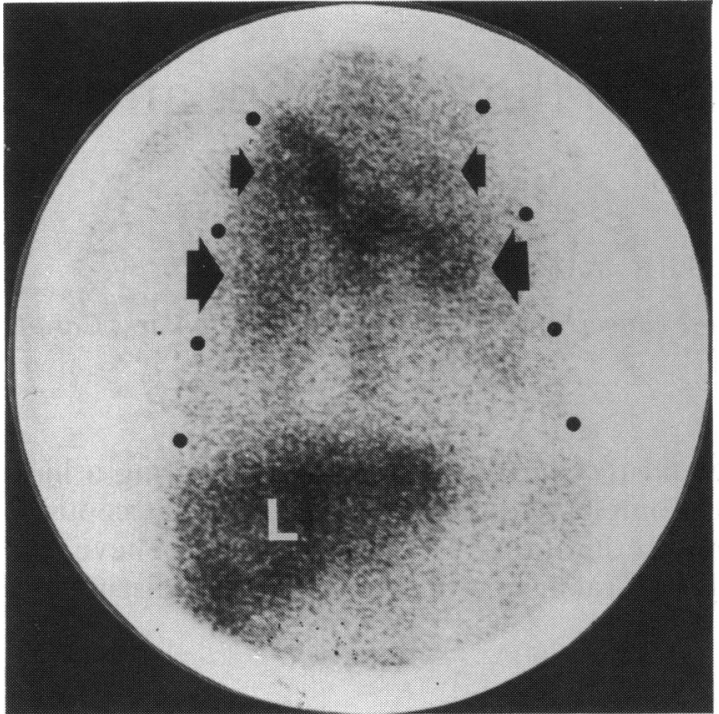

Fig. 6A

Fig. 6 Case 3. Eight months after the phase shown in Fig. 5 , soon after discontinuing prednisolone therapy an increased ${ }^{67}$ Ga uptake was measured (A) in the lungs (grade 3) (arrows). Thoracic contours are marked with dots. L=liver. The uptake over the orbits and parotids was unchanged, although uveitis became reactivated and $a(\mathrm{~B})$ fluorescein iris angiogram showed sarcoid nodules in the left iris (arrows).

more often than is suspected on the basis of ocular and parotid symptoms. Moreover, Heerfordt's disease, earlier regarded as a rare manifestation of sarcoidosis, ${ }^{11} 1$ has been brought to a new focus by the gallium examinations.

\section{References}

1 Beaumont D, Herry JY, Sapene M, Bourguet P, Larzul JJ, De Labarthe B. Gallium-67 in the evaluation of sarcoidosis: correlations with serum angiotensin-converting enzyme and bronchoalveolar lavage. Thorax 1982: 37: 11-8.

2 Koegh BA. Hunninghake GW, Line BR, Crystal RG. The alveolitis of pulmonary sarcoidosis. Evaluation of natural history and alveolitis-dependent changes in lung function. Am Rev Respir Dis 1983; 128: 256-65.

3 Heshiki A, Schatz SL, McKusick KA. Bowersox DW, Soin JS Wagner HN. Gallium 67 citrate scanning in patients with pulmonary sarcoidosis. AJR 1974: 122: 744-9.

4 Klech H, Köhn H, Kummer F. Mostbeck A. Assessment of activity in sarcoidosis. Sensitivity and specificity of ${ }^{67}$ gallium scintigraphy, serum ACE levels, chest roentgenography, and blood lymphocyte subpopulations. Chest 1982; 82: 732-8.

5 Lieberman J, Schleissner LA, Nosal A. Sastre A, Mishkin FS. Clinical correlations of serum angiotensin-converting enzyme (ACE) in sarcoidosis. A longitudinal study of serum ACE, ${ }^{67}$ gallium scans. chest roentgenograms, and pulmonary function. Chest 1983: 84: 522-8

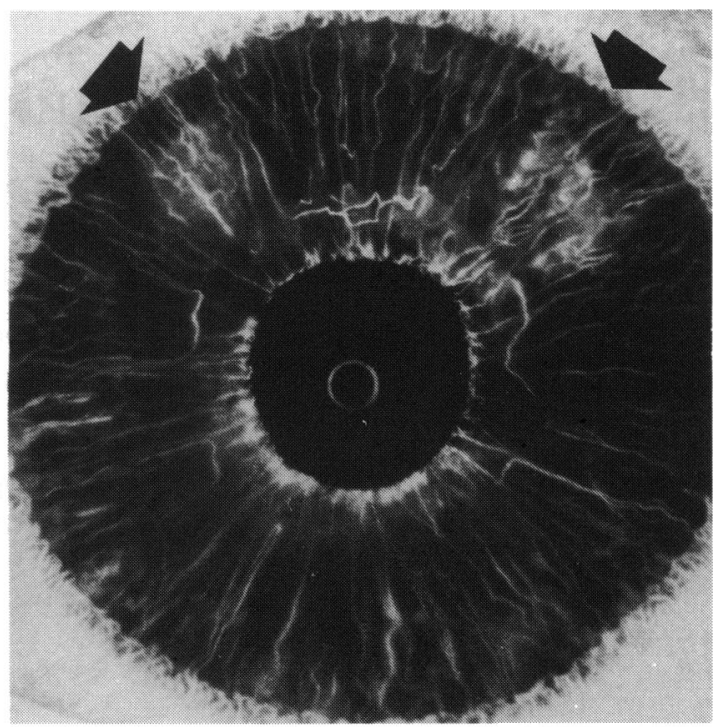

Fig. 6B

6 Weinreb RN, Barth R, Kimura SJ. Limited gallium scans and angiotensin converting enzyme in granulomatous uveitis. Ophthalmology (Rochester) 1980; 87: 202-6.

7 Wcinreb RN, Yavitz EQ, O'Connor GR, Barth RA. Lacrimal gland uptake of gallium citrate Ga 67. Am J Ophthalmol 1981; 92: 16-20.

8 Karma A, Virtanen PJ, Torniainen PJ, Ruokonen AO. Gallium67 scanning in ocular sarcoidosis. In: Saari KM, ed. Uveitis update. Amsterdam: Elscvier, 1984: 449-53.

9 Karma A, Poukkula A, Ruokonen A. Gallium ${ }^{67}$ citrate scanning in patients with lacrimal gland and conjunctival sarcoidosis. A report on threc cases. Acta Ophthalmol (Kbh) 1984; 62: 549-55.

10 Karma A. Conjunctival biopsy in sarcoidosis. In: Henkind P, ed. Acta: XXIV International Congress of Ophthalmology. Philadelphia: Lippincott, 1983.

11 Nosal A, Schleissner LA, Mishkin FS, Lieberman J. Angiotensin-I-converting enzyme and gallium scan in noninvasive evaluation of sarcoidosis. Ann Intern Med 1979; 90: $328-31$

12 Edwards CL, Hayes RL. Tumor scanning with ${ }^{67} \mathrm{Ga}$ citrate. J Nucl Med 1969; 10: 103-5.

13 Staab EV, McCartney WH. Role of gallium 67 in inflammatory disease. Semin Nucl Med 1978; 8: 219-34.

14 Mishkin FS, Maynard WP. Lacrimal gland accumulation of ${ }^{67} \mathrm{Ga}$. J Nucl Med 1974; 15: 630-1.

15 Karma A. Ophthalmic changes in sarcoidosis. Acta Ophthalmol (Kbh) 1979; suppl 141: 1-94.

16 Crick RP, Hoyle C, Smellic H. The cyes in sarcoidosis. $\mathrm{Br} \mathrm{J}$ Ophthalmol 1961; 45: 461-81.

17 Dijkstra PF, Alberts C. Sialographic characteristics in sarcoidosis. Eur J Respir Dis 1984; 65: 109-13.

18 Hecrfordt CF. Über eine 'Febris uveo-parotidea subchronica', an der Glandula Parotis und der Uvea des Auges lokalisiert und häufig mit Paresen cerebrospinaler Nerven kompliziert. Graefes Arch Clin Exp Ophthalmol 1919; 70: 254-73.

19 Bruins Slot WJ, Goedbloed J, Goslings J. Die Besnier-Boeck(Schaumann-)sche Krankheit und die Uveo-Parotitis (Heerfordt). Acta Med Scand 1938; 94: 74-97.

Accepted for publication 15 July 1986. 